\title{
Avaliação da Acessibilidade de Sítios Web por meio de Métricas de Software
}

\author{
Sinésio T. Lima, Fernanda Lima, Káthia M. De Oliveira \\ Universidade Católica de Brasília \\ SGAN 916 Norte Av. W5 - Asa Norte - 70.790-160 - Brasília - DF - Brazil \\ stlima@gmail.com, \{ferlima, kathia\}@ucb.br
}

\begin{abstract}
The Internet experienced several advances that allowed global access to increasing levels of information in diverse formats and contents. However, people with visual disabilities face many accessibility barriers that hinder the adequate understanding of Web contents. In Brazil, the Federal Government possesses an initiative, via Decree Law, that stipulates the mandatory accessibility to Governmental Web sites content. In this context, it is necessary to define how to evaluate accessibility to support the quality of these sites with respect to this aspect. Therefore, this article proposes a means of Web accessibility evaluation through software metrics.
\end{abstract}

Resumo. A Internet vivenciou inúmeros avanços que permitiram o acesso global a crescentes níveis de informação em diversos formatos e conteúdos. No entanto, pessoas com inabilidades visuais enfrentam muitas barreiras de acessibilidade que impedem a adequada compreensão dos conteúdos dos sítios Web. No Brasil, o Governo Federal possui uma iniciativa, via Decreto Lei, que prevê a obrigatoriedade da acessibilidade do conteúdo dos sítios Web Governamentais. Nesse contexto, é necessário definir como avaliar a acessibilidade para apoiar a garantia da qualidade desses sítios no que se refere a este aspecto. Este artigo propõe, portanto, como avaliar a acessibilidade Web por meio de métricas de software.

\section{Introdução}

As organizações têm se apoiado na tecnologia Web para tornar conhecidos seus produtos e serviços, atrair e fidelizar clientes. A Internet tornou-se vitrine para a maioria das empresas e também campo fértil para a disseminação de informações em larga escala, propiciando a utilização de seus recursos por milhões de pessoas no mundo inteiro. No entanto, o conteúdo dos sítios Web não é universalmente acessível e, desta forma, a interação das pessoas com inabilidades visuais com os sítios Web é dificultada devido à existência de barreiras de acessibilidade que impedem a correta interpretação do conteúdo Web por parte destes usuários.

A qualidade dos sítios Web está ligada a diversos fatores mensuráveis: desempenho, disponibilidade, navegabilidade, facilidade de uso, atratividade, acessibilidade etc. A acessibilidade dos conteúdos Web, de forma isolada, é um importante componente de qualidade que deve ser observado no conjunto de serviços providos pelos mantenedores de sítios Web. Essa importância é explicitada com a 
assinatura, no Brasil, do Decreto Lei 5.296 que obriga a adequação dos conteúdos dos sítios do Governo às regras de acessibilidade Web vigentes.

Por outro lado, é importante definir como avaliar a acessibilidade de forma que seja possível a elaboração de melhores contratos de software entre cliente e provedores. Nesse aspecto, acordos de nível de serviço ou SLAs (Service Level Agreements) são utilizados para estabelecer padrões mínimos de qualidade aos serviços prestados pelos provedores aos seus clientes [Office for Government Commerce 2001]. O principal componente de um SLA é o catálogo de serviços no qual são definidos, entre outros itens, fatores mensuráveis, ou indicadores, que darão aos clientes a visibilidade acerca do cumprimento dos níveis de serviço especificados. Neste contexto, este artigo propõe definir como avaliar a acessibilidade por meio de métricas de software que possam, posteriormente, ser utilizadas na definição de indicadores para um catálogo de serviços de SLAs de acessibilidade Web.

Nas próximas seções são apresentados: Os conceitos da acessibilidade Web e as iniciativas existentes (seção 2), os métodos para a avaliação da acessibilidade Web encontrados na literatura (seção 3), a abordagem para a avaliação da acessibilidade proposta neste trabalho (seção 4) e sua aplicação (seção 5). Na seção 6 são apresentadas as conclusões do trabalho.

\section{Acessibilidade Web}

Acessibilidade Web significa que pessoas com inabilidades visuais, auditivas, físicas, da fala, cognitivas ou neurológicas podem perceber, entender, navegar e interagir com a Web [Henry et al. 2005]. A percepção é definida como a aquisição de conhecimento por intermédio dos sentidos. O entendimento é definido como a faculdade de entender, a razão, compreensão e inteligência. A interação é definida como a ação que se exerce mutuamente entre duas ou mais coisas, ou entre duas ou mais pessoas [Novo Dicionário Aurélio 2006].

A Iniciativa de Acessibilidade Web ou WAI (Web Accessibility Initiative), patrocinada pelo consórcio $\mathrm{W} 3 \mathrm{C}$, estabelece um conjunto de guias de acessibilidade que devem ser utilizadas pelos desenvolvedores Web como padrão para o desenvolvimento de conteúdos Web acessíveis. O guia WCAG (Guia de Acessibilidade para Conteúdos Web) se destaca por constituir o conjunto oficial de regras para a acessibilidade Web [Chisholm, Vanderheiden and Jacobs 1999a]. O WCAG é composto por 14 diretrizes gerais para a acessibilidade Web. Cada diretriz é composta por pontos de verificação que explicam aspectos específicos da acessibilidade e sua respectiva aplicação. Cada ponto de verificação possui uma prioridade associada que indica sua criticidade quanto à implementação dos conteúdos acessíveis. Os pontos de verificação (PV) de prioridade 1 devem obrigatoriamente ser implementados. Os PVs de prioridade 2 devem ser implementados quando possível, para melhoria da acessibilidade. Os PVs de prioridade 3 podem ou não ser implementados, sem prejuízo da acessibilidade [Chisholm, Vanderheiden and Jacobs 1999b].

O guia WCAG está atualmente na versão 1.0 que pode ser referenciada oficialmente pelos desenvolvedores Web [Henry et al. 2006b]. A versão 2.0 está sendo desenvolvida para atender às várias tecnologias Web que foram lançadas desde a versão 
1.0, em 1999, e visa garantir maior facilidade de uso e precisão quanto à avaliação dos sítios Web [Henry et al. 2007].

Adicionalmente, a versão 2.0 aprimorou alguns conceitos em relação à versão anterior. Nessa versão, os pontos de verificação e as prioridades passaram a ser referenciados como critérios de sucesso e níveis, respectivamente. No entanto, as principais inovações estão contidas no maior detalhamento das diretrizes e sua aplicabilidade, adição de novas diretrizes e agrupamento destas em princípios de acessibilidade. Nesta versão são considerados quatro princípios básicos: (1) o conteúdo deve ser perceptível, (2) os componentes de interface devem ser operáveis, (3) o conteúdo e controles devem ser entendíveis, (4) o conteúdo deve ser robusto para interagir com os agentes de usuário. Cada princípio contém diretrizes gerais que são organizadas em níveis e critérios de sucesso [Chisholm, Slatin and Gregg 2007].

A versão 1.0 do WCAG é composta por 14 diretrizes, mais de 70 pontos de verificação e 3 prioridades possíveis, enquanto que a versão 2.0 é composta por 4 princípios, 13 diretrizes, mais de 50 critérios de sucesso e 3 níveis de acessibilidade. Comparativamente, os conceitos de nível e critério de sucesso, no WCAG 2.0, são equivalentes aos conceitos de prioridade e pontos de verificação, respectivamente, para o WCAG 1.0.

Para conciliar as inovações propostas pela nova versão, o consórcio W3C definiu um mapeamento entre as duas versões. O mapeamento relaciona os princípios, diretrizes, níveis e critérios de sucesso da versão $2.0 \mathrm{com}$ as diretrizes, prioridades e pontos de verificação da versão 1.0. A Tabela 1 apresenta parte do conjunto de itens que foram mapeados entre as duas versões do WCAG. Analisando o mapeamento completo [Caldwell et al., 2006], é possível identificar que alguns pontos de verificação estão associados a um único critério de sucesso e que algumas diretrizes WCAG 1.0, de diferentes prioridades, foram mapeadas para diretrizes WCAG 2.0 de nível 1, indicando um agrupamento de pontos de verificação e diretrizes, ambos reduzidos no WCAG 2.0.

No Brasil, o Departamento de Governo Eletrônico produziu o documento "Recomendações de Acessibilidade para a Construção e Adaptação de Conteúdos do Governo Brasileiro na Internet", cujo conteúdo referencia o WCAG 1.0. O documento é um modelo de acessibilidade que também é chamado eMAG (Modelo de Acessibilidade de Governo Eletrônico). O eMAG foi produzido a partir de um estudo comparativo das normas de acessibilidade adotadas em diversos países (Section 508 (EUA), Common Look and Feel for The Internet (CANADÁ), National Disability Authority (Irlanda), entre outros) e o WAI/W3C - Web Accessibility Initiative [Brasil 2005; Section 508 1998; Treasury Board of Canada Secretariat 2004; National Disability Authority 2006]. 
Tabela 1 - Mapeamentos entre guias WCAG 1.0 e WCAG 2.0.

\begin{tabular}{|c|c|c|c|c|c|c|c|c|c|}
\hline 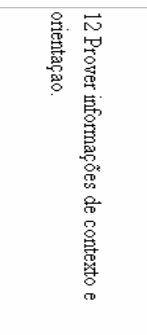 & 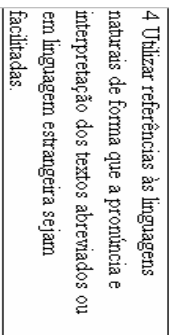 & 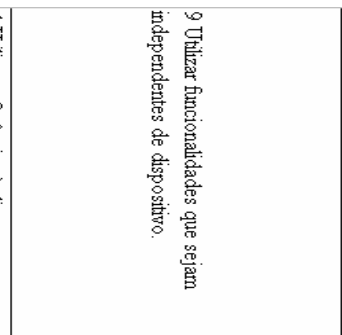 & 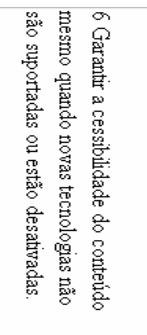 & 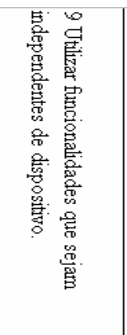 & 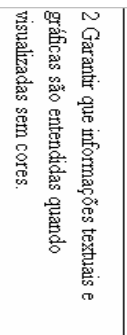 & 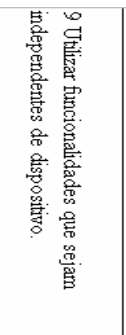 & 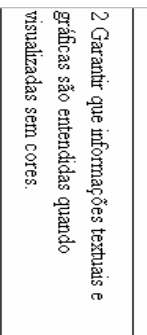 & 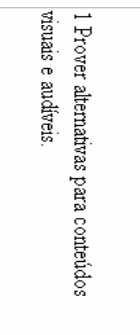 & 䓲 \\
\hline 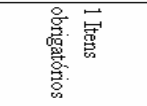 & 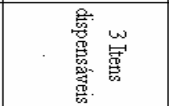 & \multicolumn{4}{|c|}{ 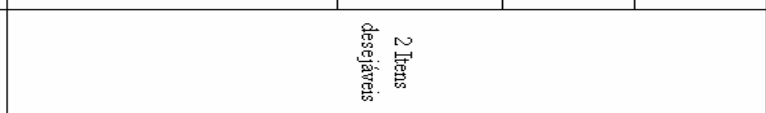 } & \multicolumn{3}{|c|}{ 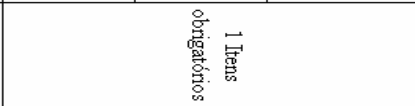 } & \\
\hline 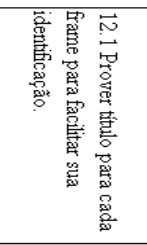 & 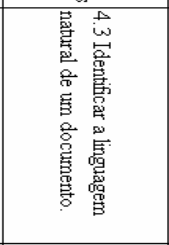 & 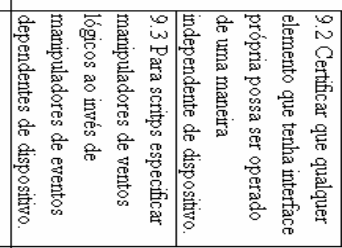 & 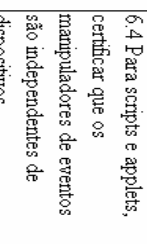 & 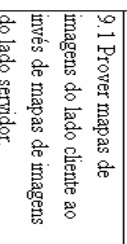 & 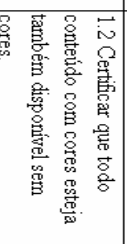 & 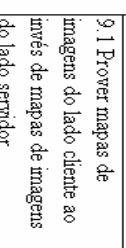 & 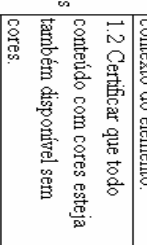 & 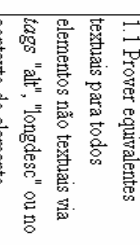 & 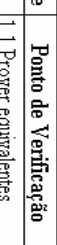 \\
\hline 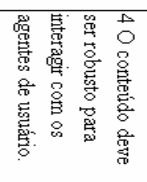 & 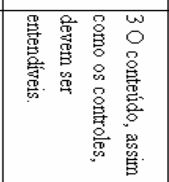 & $x_{1}$ & 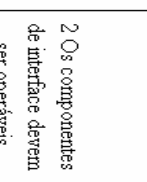 & & & \multicolumn{3}{|c|}{ 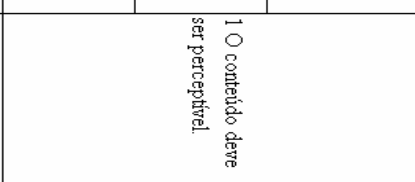 } & 櫓: \\
\hline 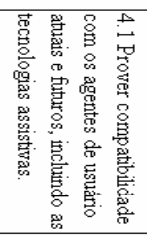 & 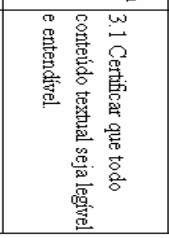 & & 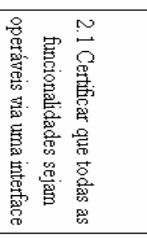 & & & \multicolumn{3}{|c|}{ 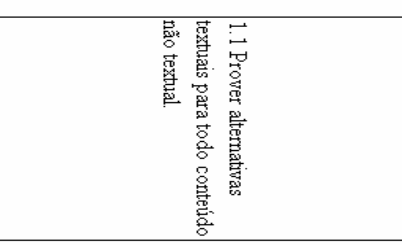 } & 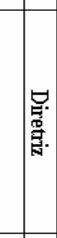 \\
\hline \multicolumn{9}{|c|}{ 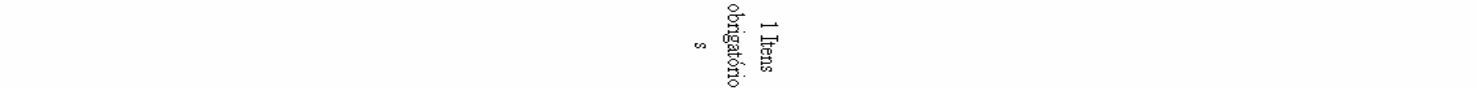 } & 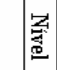 \\
\hline 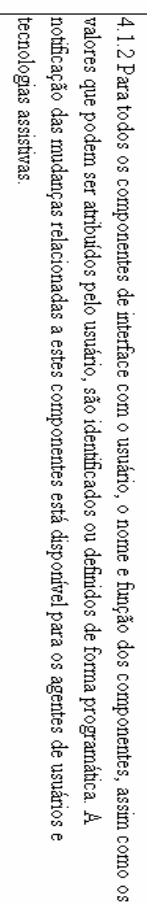 & 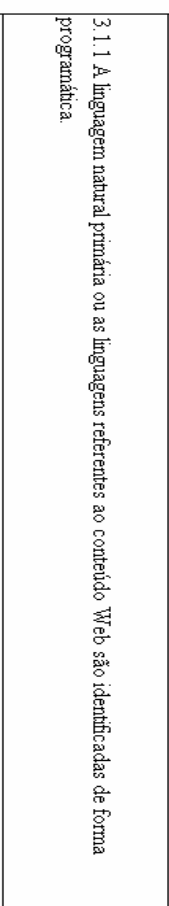 & & 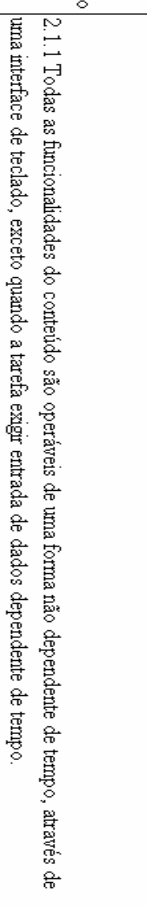 & & & & 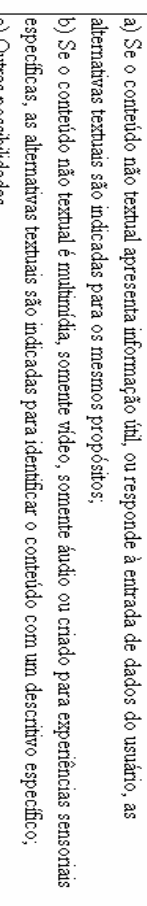 & & | \\
\hline
\end{tabular}




\section{Métodos para a Avaliação da Acessibilidade Web}

Vários autores abordam a avaliação da acessibilidade como fator preponderante para a melhoria da qualidade dos conteúdos dos sítios Web. Alguns autores [Chisholm, Vanderheiden and Jacobs 1999b; Mankoff, Fait and Tran 2005] enfocam mecanismos de avaliação, enquanto outros [Parmanto and Zeng 2005; Hackett, Parmanto and Zeng 2003] propõem métricas para avaliar níveis de acessibilidade.

Henry (2006a) aborda alguns métodos para a avaliação da acessibilidade Web: (i) avaliação heurística, ou confrontação de conformidade, que é realizada por especialistas em acessibilidade, (ii) interações nas fases de projeto de sítios (design walkthroughs), que buscam identificar problemas de acessibilidade precocemente com a ajuda de facilitadores que se passam por usuários reais, (iii) técnicas de seleção (screening techniques) utilizadas para identificar problemas de acessibilidade específicos. Nesta técnica são utilizados mecanismos de simulação de inabilidades em usuários comuns, (iv) testes formais de usabilidade que são utilizados com usuários com inabilidades reais em situações controladas. O planejamento dos testes de usabilidade envolve a escolha dos participantes, baseada em critérios específicos, a preparação de materiais de apoio, a escolha do local, o planejamento do tempo a ser alocado nos testes e a convocação dos participantes.

Melo, Baranauskas e Bonilha (2004) propuseram o método da observação participativa para a avaliação da acessibilidade com a presença de usuários reais com inabilidades. Tal método consiste em sessões de avaliação com participação de um usuário e de um observador. O usuário é convidado pelo observador para a execução de tarefas específicas envolvendo a busca de informações em alguns sítios Web. Tais tarefas são escolhidas com base em critérios para o caso específico de cada avaliação.

Durante a execução das tarefas, o usuário poderá adotar estratégias que lhe sejam mais convenientes. O observador deverá interagir com o usuário a fim de fornecer-lhe o entendimento correto do objetivo a ser alcançado para cada tarefa. Durante a interação, o observador poderá estabelecer um diálogo para registro das dificuldades e estratégias adotadas pelo usuário durante a execução das tarefas ou, a seu critério, e em sendo permitido pelo usuário, poderá registrar a sessão interativa em áudio e vídeo. O objetivo da sessão interativa é a identificação das barreiras encontradas e as estratégias utilizadas pelo usuário para contorná-las [Melo, Baranauskas and Bonilha 2004].

Finalmente, Abou-Zahra (2006) propõe diferentes abordagens para a avaliação da acessibilidade Web: (i) por intermédio de revisão preliminar para identificação de potenciais violações, (ii) considerando a conformidade com o guia de acessibilidade de conteúdos Web (WCAG 1.0), (iii) utilizando abordagens para contextos específicos envolvendo a fase de desenvolvimento dos sítios Web, avaliação de sítios legados e avaliação de páginas com conteúdo dinâmico, (iv) com o envolvimento de usuários reais com inabilidades durante o desenvolvimento dos sítios, (v) com o uso de ferramentas automáticas, e (vi) de forma mista envolvendo revisão preliminar e equipe com experiência no guia WCAG. 


\section{Avaliação da Acessibilidade Web}

Para definir o que seria avaliado, foi utilizada a abordagem GQM - Goal Question Metric [Basili, Caldiera and Rombach 1994], pois sua aplicação permitiu identificar as principais metas da avaliação e as questões relevantes, sob o ponto de vista da percepção do usuário, em relação à acessibilidade Web. Além disso, o GQM auxiliou a definição das métricas que possibilitaram mensurar quantitativa e qualitativamente a acessibilidade das tarefas avaliadas.

O GQM é baseado na premissa de que, quando se pretende medir algo, é necessário, primeiramente, definir as metas ou objetivos que se pretende alcançar. Uma vez definidos os objetivos, deve-se estabelecer as questões-chave que os descrevam e, posteriormente, definir meios que possibilitem medir o cumprimento de tais objetivos [Basili, Caldiera and Rombach 1994]. Isto significa que alguns objetivos (Goals), que servirão de guia para a elaboração de questões (Questions), devem ser identificados e especificados de modo preciso. As questões, por sua vez, orientarão a definição de métricas (Metrics) em um determinado contexto para o objeto estudado.

O objetivo da utilização do GQM para este trabalho, de acordo com o formato proposto por Soligen e Berghout (1999), é descrito abaixo:

\section{Analisar sítios Web, \\ Com o propósito de avaliar a qualidade dos conteúdos Web, Com respeito à acessibilidade, \\ Sob ponto de vista de usuários com inabilidades, No contexto do Governo Federal.}

Para definir o que seria avaliado, foi realizada uma investigação sobre os conceitos existentes sobre a acessibilidade Web. De acordo com as definições apresentadas na seção 2, foi possível identificar que a avaliação da acessibilidade significa, sobretudo, avaliar a capacidade do usuário em perceber, operar e entender o conteúdo dos sítios Web. Dessa forma, as seguintes questões foram definidas para avaliar a acessibilidade sob o ponto de vista de usuários com inabilidades:

- Qual o grau de percepção do conteúdo dos sítios Web investigados?

- Qual a facilidade de operação do conteúdo dos sítios Web?

- Qual o grau de entendimento do conteúdo dos sítios Web?

No entanto, existe uma preocupação, associada à acessibilidade, que remete à capacidade do usuário em alcançar o objetivo desejado com um esforço aceitável e de forma satisfatória. Esse é um aspecto explorado pela ISO/IEC 9126 [International Organization for Standardization 2001] no que diz respeito à qualidade em uso, ou seja, a capacidade de um produto de software em garantir que os usuários consigam atingir metas específicas com eficácia, produtividade segurança e satisfação, em contextos de uso específicos.

No caso deste trabalho, o objetivo dos usuários será a execução de tarefas específicas durante as sessões de interação em alguns sítios Web do Governo Federal. Essa situação permitiu o desenvolvimento de três novas questões: 
- Qual a eficácia dos usuários na execução de tarefas específicas durante a interação com os sítios Web investigados?

- Qual a produtividade dos usuários na execução de tarefas específicas durante a interação com os sítios Web?

- Qual o grau de satisfação dos usuários na execução de tarefas específicas durante a interação com os sítios Web?

Para responder as três primeiras questões foram definidas métricas de acordo com os princípios de acessibilidade do guia WCAG. Dessa forma, para coletar os valores das métricas foi utilizada a ferramenta automatizada TAW StandAlone [Fundación CTIC 2005].

A ferramenta automatizada foi utilizada para coletar os quantitativos de violações aos pontos de verificação de acessibilidade do WCAG 1.0. Os quantitativos foram extraídos dos relatórios gerados pela ferramenta e consolidados para cada tarefa executada. No entanto, para obter os quantitativos relacionados aos princípios da acessibilidade, foi necessário realizar o mapeamento das versões 1.0 e 2.0 do WCAG, visto que a ferramenta automatizada é baseada na versão 1.0 e os princípios são definidos na versão 2.0. Em outras palavras, o mapeamento permitiu identificar quais pontos de verificação (WCAG 1.0) se relacionavam com quais critérios de sucesso (WCAG 2.0).

Para a definição das métricas, além da ferramenta automatizada, também foi utilizado um questionário psicométrico (Figura 1) cujo objetivo é a captura da percepção do usuário em relação aos princípios da acessibilidade. O questionário possui questões subjetivas cujas respostas são coletadas utilizando uma escala de valores de Likert variando de 0 (para o pior caso) a 7 (para o melhor caso). O questionário, apresentado na Figura 1, é composto de três partes: (1) dados demográficos, (2) dados da observação e (3) dados da percepção do usuário.

Os dados demográficos servem para descrever características específicas da população de usuários, que avaliaram a acessibilidade dos sítios Web. Algumas dessas características incluem o nível de deficiência visual, a experiência no uso da Web e a opinião dos usuários em relação à acessibilidade da Web em um contexto geral. Os dados da observação são coletados por um observador externo que interage com os usuários em todas as sessões de avaliação. O observador externo é o responsável por coletar os dados relacionados à correta finalização das tarefas pelos usuários e o tempo médio gasto por estes para concluir cada tarefa.

Como parte do conjunto de dados coletados por meio do questionário psicométrico, os dados da percepção do usuário têm grande relevância devido à sua coleta ser realizada por meio da prática real dos usuários nas sessões de interação com os sítios Web. Para o contexto da avaliação da acessibilidade, o cenário ótimo considera as métricas com valores tendendo a zero, para os números de violações (métricas $2,7 \mathrm{e}$ 9), valores tendendo a $100 \%$ para os percentuais (métricas 11 e 12) e valores tendendo a sete para os níveis (graus) de Percepção, Operação e Entendimento dos conteúdos Web (métricas 1, 3, 4, 5, 6, 8 e 10). As métricas definidas são apresentadas na Tabela $2 \mathrm{e}$ serão utilizadas no contexto da melhoria da qualidade da acessibilidade dos conteúdos Web. 
Tabela 2 - Métricas definidas para as questões GQM.

\begin{tabular}{|c|c|c|c|c|c|c|c|c|c|c|c|c|}
\hline 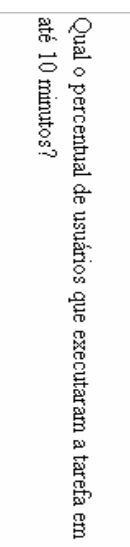 & 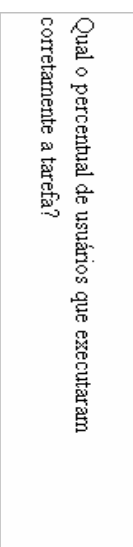 & 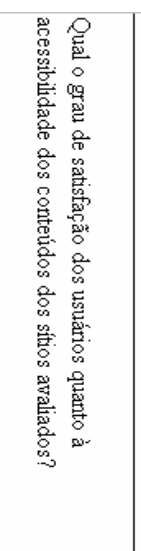 & 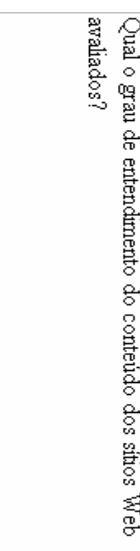 & & \multicolumn{5}{|c|}{ 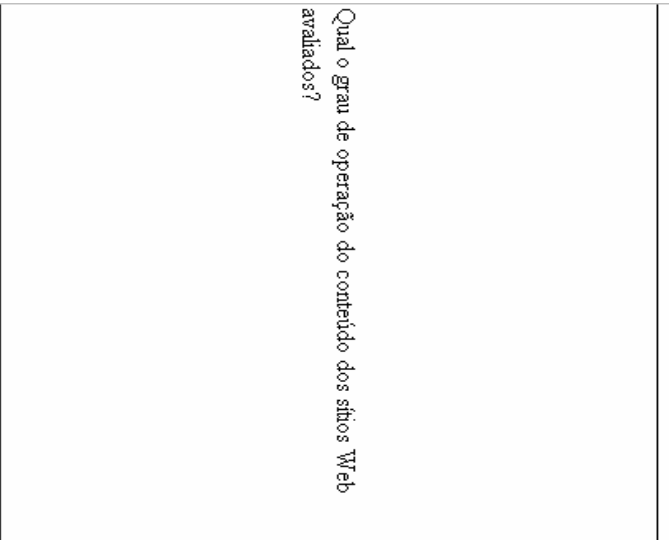 } & \multicolumn{2}{|c|}{ 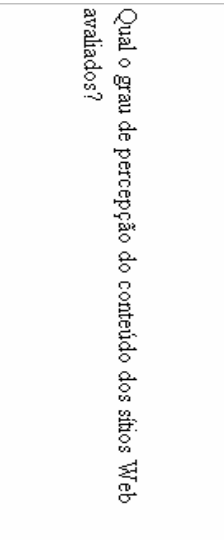 } & 萝 \\
\hline 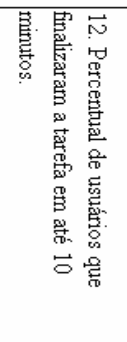 & 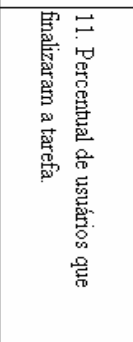 & 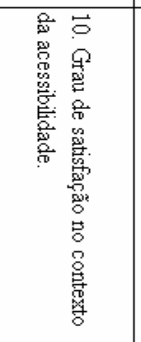 & 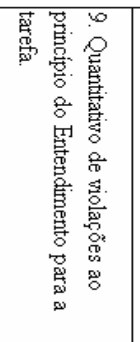 & 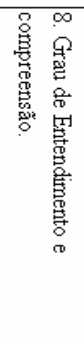 & 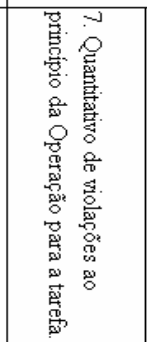 & 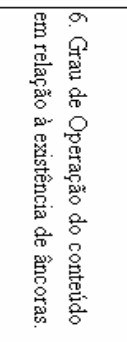 & 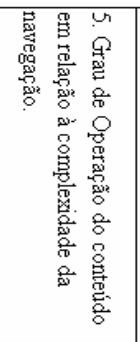 & 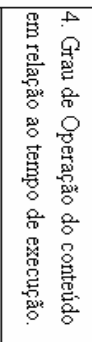 & 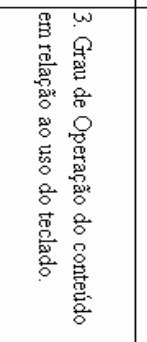 & 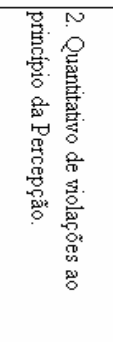 & 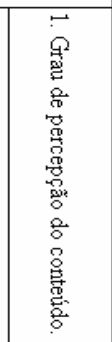 & 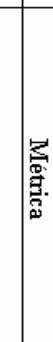 \\
\hline 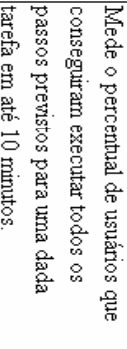 & 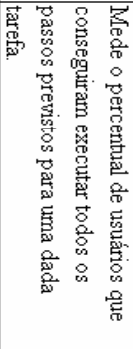 & 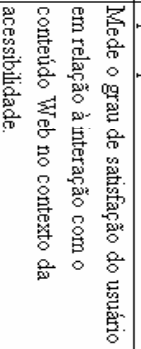 & 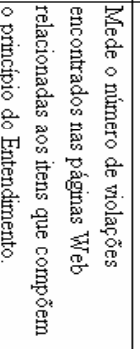 & 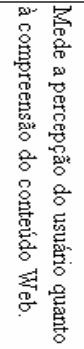 & 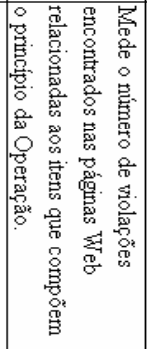 & 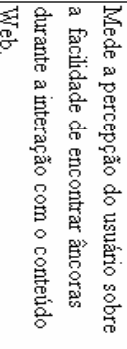 & 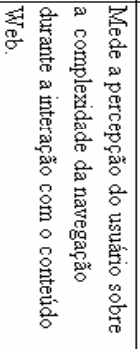 & 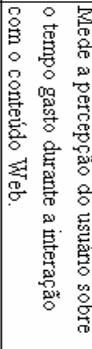 & 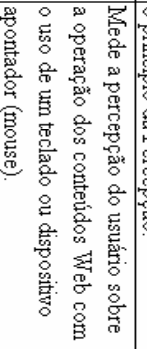 & 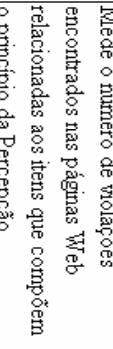 & 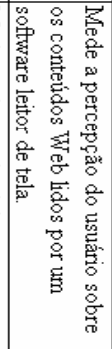 & 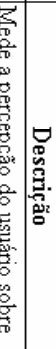 \\
\hline 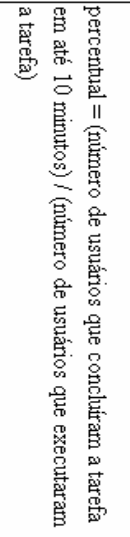 & 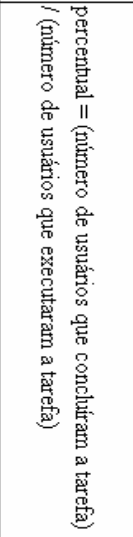 & 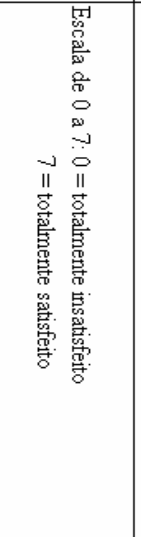 & 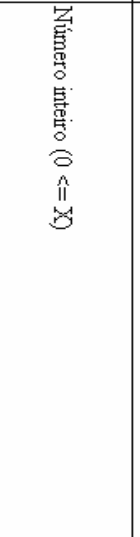 & 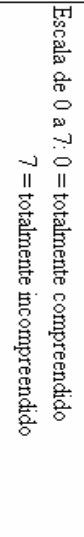 & 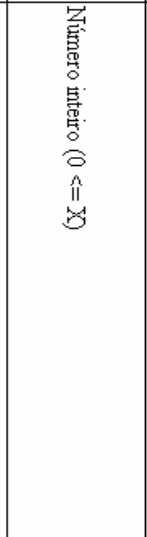 & 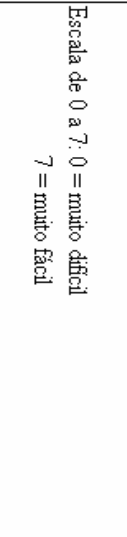 & 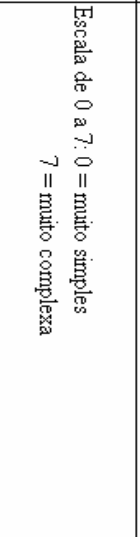 & 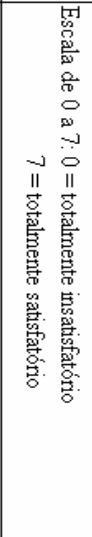 & 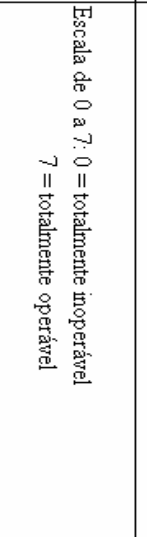 & 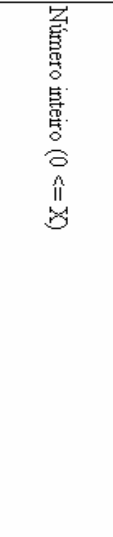 & 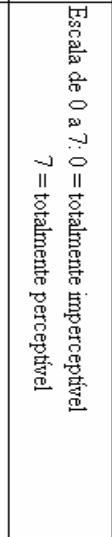 & . \\
\hline 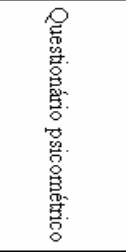 & 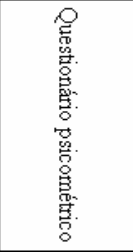 & 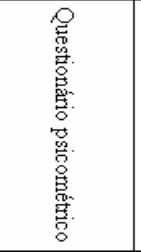 & 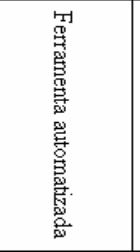 & 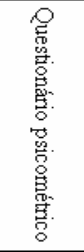 & 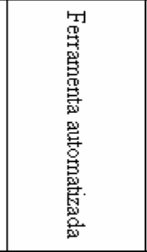 & 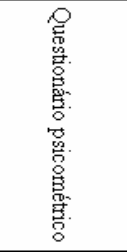 & 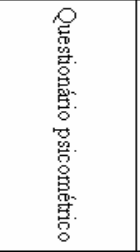 & 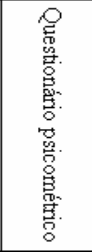 & 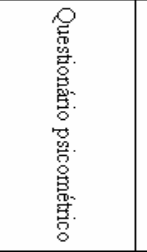 & 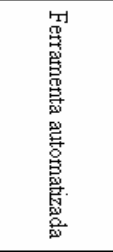 & 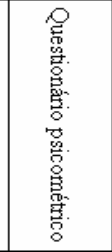 & 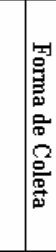 \\
\hline
\end{tabular}


Figura 1 - Questionário psicométrico.

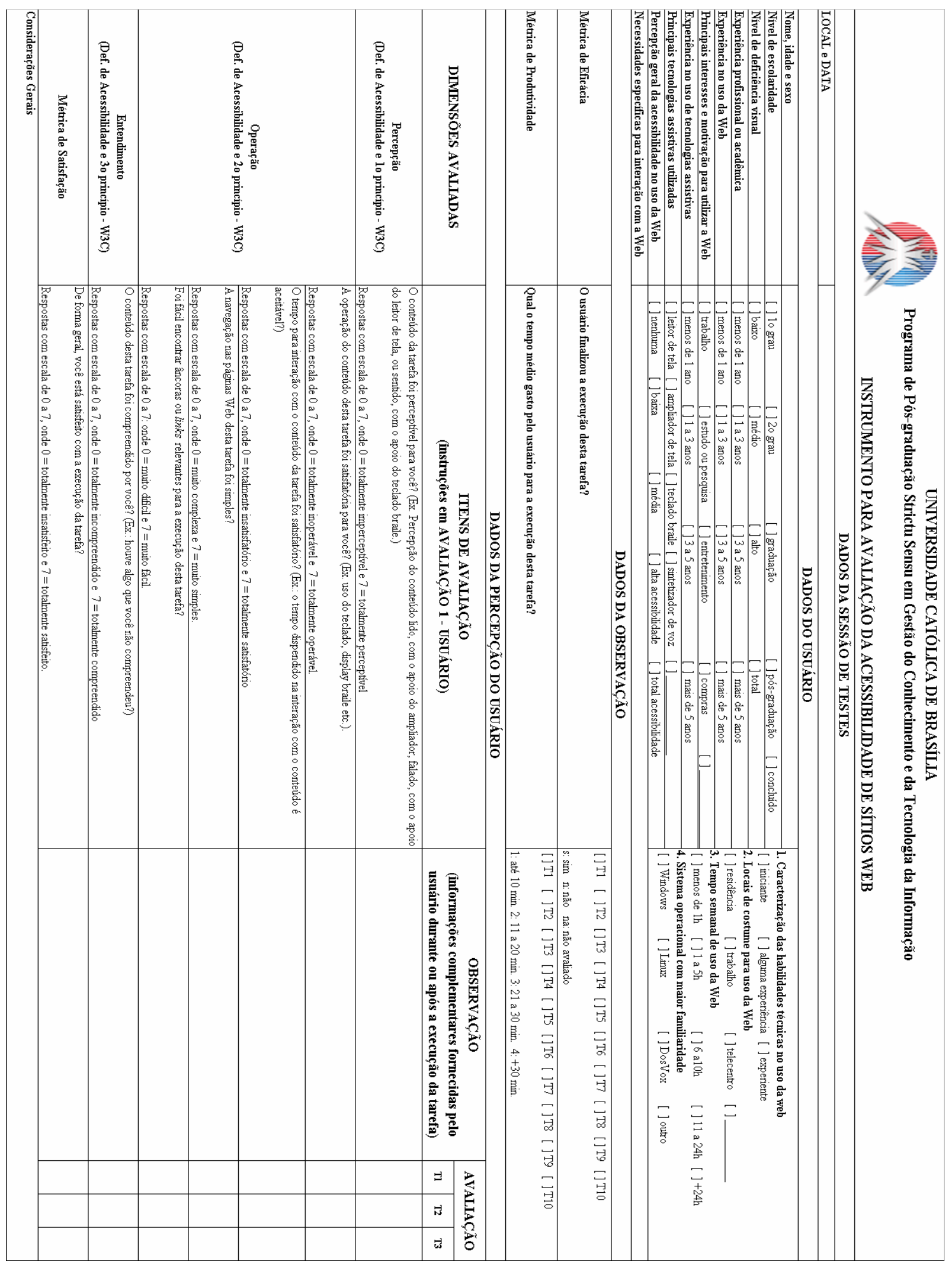




\section{Aplicação Prática}

A escolha dos sítios investigados foi realizada utilizando os resultados publicamente disponíveis no sítio do Prêmio iBEST que é referência, no Brasil, para a premiação dos melhores sítios da Internet brasileira, segundo critérios estabelecidos por especialistas em Internet e comércio eletrônico. Os sítios escolhidos foram extraídos da categoria CIDADANIA e da subcategoria GOVERNO que engloba os sítios de prestação de serviços governamentais que obtiveram a maior votação. Foram escolhidos três sítios de serviços públicos: Secretaria da Receita Federal (www.receita.fazenda.gov.br), Previdência Social (www.previdencia.gov.br) e Instituto Brasileiro de Geografia e Estatística - IBGE (www.ibge.gov.br).

Para cada sítio foi identificada uma tarefa específica com o objetivo de busca de informações no sítio alvo. A escolha das tarefas obedeceu critérios que incluíram níveis de complexidade. As tarefas, e seus respectivos objetivos, são os seguintes, considerando a ordem dos sítios citada acima:

- Tarefa 1 (T1): Dentre os serviços disponíveis para pessoa física, consultar informações referentes à restituição do imposto de renda, para um dado CPF, para o ano 2006;

- Tarefa 2 (T2): Identificar quais documentos são necessários para o serviço de inscrição na Previdência Social;

- Tarefa 3 (T3): Encontrar a página do serviço “Fale Conosco" e identificar quais assuntos podem ser tratados por e-mail.

A execução das tarefas foi realizada em sessões de avaliação com a participação de dez usuários com níveis de deficiência visual e experiência com a Web diferenciados. Todas as sessões foram conduzidas em ambientes com disponibilidade dos seguintes recursos: computador pessoal com teclado, mouse e caixas de som, navegador Web Internet Explorer ou Firefox Mozilla e os softwares leitores de tela Jaws ou Virtual Vision.

A maioria das sessões de avaliação foi registrada em vídeo, com a devida autorização dos usuários, para posterior consulta e para garantir a fidelidade do processo de avaliação em relação aos depoimentos dos usuários quanto à acessibilidade das tarefas. O tempo médio gasto em cada sessão de avaliação foi de duas horas. Este parâmetro foi influenciado diretamente pelo grau de detalhamento dos comentários feitos por cada usuário, por sua experiência no uso da Web e por sua disponibilidade.

A preparação de cada sessão de avaliação obedeceu uma ordem específica de passos descritos a seguir:

- Abordagem inicial do avaliador para explicação sucinta sobre a pesquisa, motivação e objetivos;

- Detalhamento dos objetivos da sessão de avaliação;

- Explicação sucinta sobre o conteúdo do questionário psicométrico;

- Fornecimento de tempo para o avaliador configurar o computador ou softwares de acordo com suas preferências;

- Coleta de dados demográficos e preenchimento destes no questionário psicométrico; 
- Coleta da autorização para registro da sessão em vídeo;

- Leitura em voz alta do título, URL inicial e objetivo de cada tarefa específica;

- Observação da interação do usuário com o sítio e participação durante a interação.

Após finalização da coleta de dados, foram atribuídos os valores para as métricas definidas anteriormente. As métricas e seus respectivos valores são apresentados na Tabela 3. Considerando os dados demográficos coletados, $60 \%$ dos usuários concluíram ou estão em fase de conclusão da graduação, $60 \%$ informaram ter alto nível de deficiência visual ou deficiência total, $80 \%$ atuam no mercado de trabalho há mais de 5 anos, $80 \%$ trabalham com a Web há mais de 5 anos, $100 \%$ utilizam a Web para estudo ou trabalho, $90 \%$ afirmaram que a acessibilidade geral da Web é baixa ou média e $70 \%$ informaram que utilizam a Web mais de vinte e quatro horas semanalmente.

Tabela 3 - Métricas de acessibilidade e valores.

\begin{tabular}{|c|c|c|c|c|c|c|}
\hline \multicolumn{2}{|r|}{ Métricas (e questões) } & \multicolumn{3}{|c|}{ Valores por tarefa } & \multirow{2}{*}{$\begin{array}{c}\text { Tarefa c/ } \\
\text { melhor } \\
\text { valor }\end{array}$} & \multirow{2}{*}{$\begin{array}{c}\text { Tarefa c/ } \\
\text { pior } \\
\text { valor }\end{array}$} \\
\hline & & $\mathrm{T} 1$ & $\mathrm{~T} 2$ & T3 & & \\
\hline \multirow{2}{*}{ Q1 } & Grau de percepção do conteúdo & 2,9 & 5,5 & 6 & $\mathrm{~T} 3$ & $\mathrm{~T} 1$ \\
\hline & Quantitativo de violações ao princípio da Percepção & 15 & 59 & 22 & $\mathrm{~T} 2$ & T1 \\
\hline \multirow{5}{*}{ Q2 } & Grau de Operação com relação ao uso do teclado & 5,4 & 5,8 & 6,2 & T3 & $\mathrm{T} 1$ \\
\hline & Grau de Operação com relação ao tempo de execução & 2,3 & 5,1 & 5,8 & T3 & $\mathrm{T} 1$ \\
\hline & Grau de Operação com relação à navegação. & 5 & 5,8 & 6 & $\mathrm{~T} 3$ & T1 \\
\hline & Grau de Operação com relação à existência de âncoras & 5,2 & 6 & 5,8 & $\mathrm{~T} 2$ & $\mathrm{~T} 1$ \\
\hline & Quantitativo de violações ao princípio da Operação & 2 & 0 & 0 & $\mathrm{~T} 2 / \mathrm{T} 3$ & $\mathrm{~T} 1$ \\
\hline \multirow{2}{*}{ Q3 } & Grau de Entendimento e compreensão & 3,3 & 5,6 & 6 & $\mathrm{~T} 3$ & T1 \\
\hline & Quantitativo de violações ao princípio do Entendimento & 5 & 4 & 0 & T3 & $\mathrm{T} 1$ \\
\hline Q4 & Percentual de Eficácia & $10 \%$ & $100 \%$ & $100 \%$ & $\mathrm{~T} 2 \sqrt{\mathrm{T}} 3$ & T1 \\
\hline Q5 & Percentual de Produtividade (até $10 \mathrm{~min}$.) & $10 \%$ & $80 \%$ & $90 \%$ & T3 & $\mathrm{T} 1$ \\
\hline Q6 & Grau de satisfação & 1 & 5,6 & 5,7 & T3 & $\mathrm{T} 1$ \\
\hline
\end{tabular}

Após a atribuição dos valores a cada métrica, as questões originais foram retomadas com o objetivo de respondê-las. As análises individuais para cada questão são apresentadas a seguir:

\section{Q1) Qual o grau de percepção do conteúdo dos sítios Web investigados?}

Em linhas gerais, os usuários obtiveram boa percepção do conteúdo das tarefas T2 e T3, visto que os resultados ficaram próximos da escala máxima (sete). Para a tarefa T1, o grau de percepção dos usuários foi sensivelmente mais baixo, indicando que o conteúdo desta tarefa não foi percebido satisfatoriamente pelos usuários. Isto pode ser explicado pelo emprego de imagens distorcidas, sem o equivalente textual ou audível, em uma das páginas que compõem a tarefa T1. Especificamente para esta tarefa, as imagens distorcidas foram utilizadas como um mecanismo de segurança que é comumente utilizado em sítios Web para evitar o roubo de informações.

As tarefas T1 e T3 obtiveram os menores quantitativos de violações para o princípio da percepção. Devido à baixa percepção dos conteúdos por parte dos usuários era esperado que o nível de violações para a tarefa $\mathrm{T} 1$ fosse o mais alto entre as três tarefas. 


\section{Q2) Qual a facilidade de operação do conteúdo dos sítios Web?}

A operação dos conteúdos, de forma geral, pode ser considerada satisfatória para todas as tarefas, à exceção da tarefa $\mathrm{T} 1$ que obteve um grau de operação, quanto ao tempo de execução, muito abaixo do registrado para as demais tarefas. Nas sessões de avaliação foi observado que os usuários perderam muito tempo na tentativa de transpor a página que continha as imagens distorcidas.

Foi observado que quanto maiores os níveis de operação dos conteúdos das tarefas, menores foram os quantitativos de violações encontrados para este princípio. Este fato indica que há uma relação inversa entre o grau de operação dos conteúdos e os quantitativos de violações, o que era esperado.

\section{Q3) Qual o grau de entendimento do conteúdo dos sítios?}

Os resultados obtidos para o grau de entendimento dos conteúdos das tarefas indicam que as imagens distorcidas também afetaram o entendimento dos conteúdos, visto que a tarefa $\mathrm{T} 1$ obteve valores muito abaixo quando comparada às demais tarefas.

Foi observado que quanto maiores os níveis de entendimento dos conteúdos das tarefas, menores foram os quantitativos de violações encontrados para este princípio. Este fato indica que também há uma relação inversa entre o grau de operação dos conteúdos e os quantitativos de violações, o que era esperado.

Q4) Qual a eficácia dos usuários na execução de tarefas específicas durante a interação com os sítios Web investigados?

Foi observado que apenas $10 \%$ dos usuários concluíram a tarefa T1 e 100\% destes concluíram as tarefas T2 e T3. Este fato é um sério indicativo de que as imagens distorcidas, sem o equivalente textual ou audível, são uma grave barreira de acessibilidade, pois é claro o impacto causado na eficácia dos usuários, durante a execução da tarefa T1.

Foi observado que, de forma geral, a eficácia foi afetada para as tarefas que obtiveram os piores níveis de percepção, operação e entendimento dos conteúdos, pois os valores atribuídos a estes princípios decrescem à medida que o nível de eficácia cai.

Q5) Qual a produtividade dos usuários na execução de tarefas específicas durante a interação com os sítios Web?

A produtividade dos usuários na execução das tarefas T2 e T3 foi bastante satisfatória, obtendo os percentuais de $80 \%$ e $90 \%$, respectivamente, para tempos de execução de até dez minutos. Dos usuários que finalizaram a tarefa T1 apenas $10 \%$ destes conseguiram executá-la em até 10 minutos. Este fato corrobora com o resultado obtido para o grau de operação com relação ao tempo de execução, visto que os valores desta métrica, coletados para a tarefa $\mathrm{T} 1$, foram significativamente inferiores quando comparados aos valores das demais tarefas.

De forma semelhante aos resultados observados para a eficácia, a produtividade também foi afetada para as tarefas que obtiveram os piores níveis de percepção, operação e entendimento dos conteúdos. Foi observado que os valores atribuídos a estes princípios decrescem à medida que a produtividade cai. 


\section{Q6) Qual o grau de satisfação dos usuários na execução de tarefas específicas durante a interação com os sítios Web?}

Durante as sessões de avaliação, os usuários foram orientados a considerar o item de satisfação como sendo um resumo das suas experiências enquanto executores das tarefas. Os resultados obtidos demonstram que a satisfação dos usuários foi diretamente afetada pelos níveis de eficácia e produtividade observados, ou seja, quanto piores os níveis de eficácia e produtividade dos usuários, pior foram os níveis de percepção, operação e entendimento relatados após a execução das tarefas. Esta relação direta também foi observada em relação à eficácia e produtividade dos usuários.

\subsection{Considerações sobre a Acessibilidade}

Os resultados demonstraram que os níveis de percepção e entendimento dos conteúdos Web são crescentes à medida que os quantitativos de violações, que afetam estes princípios, são decrescentes. Este fato permite concluir que quanto menor a quantidade de violações aos itens de verificação melhor será a acessibilidade relacionada aos princípios de operação e entendimento dos conteúdos Web.

A análise dos resultados das questões 1,2 e 3 permite concluir que a tarefa com menor grau de percepção, operação e entendimento é a tarefa T1 e estes resultados levam a crer que a presença de imagens distorcidas sem os equivalentes necessários, apenas para esta tarefa, pode ser considerada uma barreira real para a acessibilidade. De forma geral, os resultados coletados para as seis questões, também corroboram para as seguintes afirmações:

- Existe uma relação inversa entre os níveis de operação e entendimento e os quantitativos de violações obtidos para estes princípios, indicando que a acessibilidade é afetada quando há violações a estes princípios;

- Quanto menores os graus de percepção, operação e entendimento dos conteúdos Web, menores serão a eficácia, a produtividade e a satisfação dos usuários durante a interação com estes conteúdos.

De forma geral, a acessibilidade, representada pelos princípios, foi afetada pelas violações encontradas, o que está claro nos resultados comentados em cada questão. Os resultados somente foram obtidos com a aplicação da avaliação conjunta envolvendo usuários reais e a ferramenta automatizada. Portanto, a avaliação da acessibilidade Web deve considerar a participação de usuários reais e não somente a utilização de ferramentas automatizadas, visto que os quantitativos de violações, avaliados de forma isolada, pouco acrescentariam ao contexto de melhoria da qualidade dos conteúdos para os usuários de serviços Web. A obtenção das métricas qualitativas (questionário psicométrico) e quantitativas (ferramenta automatizada) foram fundamentais para o processo de avaliação, visto que a avaliação dos usuários pode indicar com segurança quais violações afetam sua interação com a Web e, desta forma, os provedores dos sítios podem estabelecer planos de ação para minimizar o impacto das violações nos sítios. 


\section{Conclusão}

A acessibilidade aos conteúdos das informações disponíveis nos sítios Web deve ser garantida a todas as pessoas, pois a interação com Web é uma necessidade crescente e, principalmente para as pessoas com inabilidades, uma forma autêntica de interação para busca de informação, entretenimento e trabalho. Neste contexto, os desenvolvedores de sítios Web devem minimizar o impacto das violações aos princípios da acessibilidade buscando eliminar barreiras de acessibilidade que mais afetam os usuários.

Este trabalho propôs como avaliar a acessibilidade Web por meio de métricas de software. A forma de avaliação proposta possibilitou avaliar a acessibilidade sob o ponto de vista de usuários com inabilidades e com o apoio de ferramenta automatizada homologada pelo consórcio W3C. Os resultados obtidos demonstraram que a acessibilidade percebida pelos usuários é diretamente impactada pela existência de violações aos pontos de verificação do WCAG 1.0 e aos critérios de sucesso do WCAG 2.0. Um fato que necessita ser mencionado é que a obrigatoriedade da acessibilidade para os sítios do Governo, prevista no Decreto Lei 5.296, ainda não foi implantada integralmente.

Estudos mais aprofundados necessitam ser realizados para complementação deste trabalho, visto que houve dificuldade para encontrar voluntários para as avaliações. Outra dificuldade encontrada, e que poderá ser minimizada em trabalhos futuros, foi a impossibilidade de avaliar mais tarefas por usuário, devido o tempo requerido para a avaliação individual de cada tarefa.

Os próximos passos visam aprimorar as análises, sobre as métricas coletadas, com o objetivo de produzir indicadores que possam ser utilizados para compor um catálogo de serviços para SLAs de acessibilidade Web. Os SLAs de acessibilidade servirão de instrumentos formais, entre desenvolvedores e clientes de conteúdos Web, para contribuir com as iniciativas de acessibilidade vigentes.

\section{Agradecimentos}

Agradecemos a todas as pessoas que participaram como voluntárias nas sessões de avaliação, e ao Telecentro Acessível de Taguatinga, cidade satélite de Brasília-DF, que colaborou com a infra-estrutura para a realização de uma sessão piloto para validação do questionário psicométrico com um de seus alunos regulares.

\section{Referências}

Abou-Zahra, S. et al. (Editors) (2006) "Evaluating Web Sites for Accessibility: Overview", http://www.w3.org/WAI/eval/Overview.html.

Basili, V. R., Caldiera, G. and Rombach, H. D. (1994) The Goal Question Metric Paradigm. Encyclopedia of Software Engineering, vol. 1, pages 528-32. John Wiley \& Sons.

Brasil. Ministério do Planejamento, Orçamento e Gestão (2005) "Modelo de Acessibilidade de Governo Eletrônico - versão 2.0.", http://www.governoeletronico.gov.br/governoeletronico.

Caldwell, B. et al. (Editors) (2006) "Comparison of WCAG 1.0 checkpoints to WCAG 2.0.”, http://www.w3.org/TR/WCAG20/appendixD.html. 
Chisholm W., Vanderheiden G. and Jacobs I. (1999a) "Checklist of Checkpoints for Web Content Accessibility Guidelines 1.0", http://www.w3.org/TR/WCAG10/fullchecklist.html.

Chisholm W., Vanderheiden G. and Jacobs I. (1999b) "Web Contents Accessibility Guidelines 1.0", http://www.w3.org/TR/WCAG10.

Chisholm W., Slatin J., Gregg and White J. (2007) "Web Contents Accessibility Guidelines 2.0", http://www.w3.org/TR/WCAG20/appendixD.html.

Fundación CTIC (2005) "Web Accessibility Test", http://www.tawdis.net/taw3/cms/en.

Hackett, S., Parmanto, B. Zeng, X. (2003) “Accessibility of Internet Websites Through Time". ACM.

Henry, S. L. et al. (Editors) (2005) "Introduction to Web Accessibility", http://www.w3.org/WAI/intro/accessibility.php.

Henry, S. L. et al. (Editors) (2006a) "Just Ask: Integrating Accessibility Throughout Design", $\quad$ http://www.w3.org/WAI/eval/users.html\#ut-access http://www.uiaccess.com/accessucd/overview.html.

Henry, S. L. et al. (Editors) (2006b) "Web Content Accessibility Guidelines (WCAG) Overview", http://www.w3.org/WAI/intro/wcag.php.

Henry, S. L. et al. (Editors) (2007) "WCAG 2 FAQ", http://www.w3.org/WAI/WCAG20/wcag2faq.

International Organization for Standardization. ISO/IEC 9126-1. Software Engineering - Product quality - Part 1: Quality model, 2001.

Mankoff, J., Fait, H. and Tran, T. (2005) "Is Your Web Page Accessible? A Comparative Study of Methods for Assessing Web Page Accessibility for The Blind". ACM. CHI'05.

Melo, A. M., Baranauskas M. C. C. and Bonilha, F.F.G. (2004) "Avaliação de acessibilidade na Web com a participação do usuário: um estudo de caso". Proceedings of the Brazilian IHC Symposium.

National Disability Authority (2006) "National Disability Authority", http://www.nda.ie.

Novo Dicionário Aurélio (2006) "Novo Dicionário Aurélio", http://200.225.157.123/dicaureliopos/login.asp.

Office for Government Commerce (2001) "ITIL - The Key to Manage IT Services: Service Delivery", Version 1.2 - Crow.

Parmanto, B. and Zeng, X. (2005) "Metric for Web Accessibility Evaluation.”, Journal for The American Society for Information Science and Technology. Vol. 56(13):1394-1404.

Section 508 (1998) "Amendment to Section 508 of the Rehabilitation Act", http://www.section508.gov/index.cfm?FuseAction=Content\&ID=14.

Soligen, R. and Berghout, E. (1999) "The Goal Question Metric Method - A Practical Guide for Quality Improvement of Software Development", Great Britain: Cambridge, McGraw-Hill.

Treasury Board of Canada (2004) "CLF for the Internet - Accessibility", http://www.tbs-sct.gc.ca/clf-nsi/inter/inter-01-tb_e.asp. 